\title{
Chorioamnionitis and Congenital Abnormalities of the Brainstem (Arcuate Nucleus and Pre-Bötzinger Complex Hypoplasia) in a Case of Late Unexplained Stillbirth
}

\author{
Rosaria Mingrone $^{1}$, Ezio Fulcheri ${ }^{2}$, Anna Maria Lavezzi ${ }^{*}, 1$ and Luigi Matturri ${ }^{1}$ \\ 1 "Lino Rossi" Research Center, Institute of Pathology, University of Milan, Italy \\ ${ }^{2}$ Department of Pathology, San Martino Hospital, University of Genoa, Italy
}

\begin{abstract}
Background: Fetal death (stillbirth), that occurs every 100-200 pregnancies in industrialized countries, has not significantly decreased in recent years, mostly because of scarce research activities in this field. Fetal death is often, in up to $60-80 \%$ of cases, unexplainable, even after routine diagnostic examinations.

Objectives: This work aims to stress the importance of in-depth histopathological investigations of both the autonomic nervous system and the placenta in sudden fetal death.

Methods: In a case of late fetal death at $40+^{3}$ weeks of pregnancy an in-depth histopathological examination of the brainstem and of the placenta was performed.

Results: The case investigated showed severe hypoplasia of the arcuate nucleus as well as of the reticular formation and particularly of the pre-Bötzinger complex in the brainstem. Pulmonary hypoplasia was also present, associated with a picture of severe (grade 3) chorioamnionitis, with inflammatory infiltrate, vasculitis of the umbilical vein and amniochorionic vessels.

Conclusions: The chorioamnionitis can allegedly contribute to stillbirth, through cytokine release, enhancing any chemoreceptor neuronal dysfunction.
\end{abstract}

Keywords: Stillbirth, unexplained fetal death, arcuate nucleus, pre-Bötzinger complex, chorioamnionitis.

\section{INTRODUCTION}

Stillbirth or fetal death is death prior to the complete expulsion or extraction from the mother of a product of conception, irrespective of pregnancy duration. Death is indicated by the fact that after such separation, the fetus does not breathe or show any other sign of life, such as beating of the heart, pulsation of the umbilical cord, or specific movements of voluntary muscles [1].

Fetal death, that occurs every $100-200$ pregnancies in industrialized countries [2], is in up to $60-80 \%$ of cases, unexplainable, even after routine diagnostic examinations [3].

Congenital abnormalities of both the autonomic nervous system and the cardiac conduction system are frequent morphological substrates of unexpected perinatal death $[4,5]$.

A common finding is the hypoplasia of the arcuate nucleus, a chemoreceptoral component of the ventral surface of the medulla oblongata, which was present in over $50 \%$ of the victims, albeit with different degrees of extension and severity [6]. Frequently, in stillbirth, the hypoplasia of the arcuate nucleus is associated with hypodevelopment of the dendritic fibers and decreased neuronal density in the pre-Bötzinger area [7], besides with hypoplasia of the parabrachial/Kölliker-Fuse complex $[8,9]$.

*Address correspondence to this author at the Institute of Pathology, University of Milan, Via della Commenda, 19, 20122 Milan, Italy; Tel: +39-0250320821; Fax: +39-02-50320823; E-mail: anna.lavezzi@unimi.it
All these nuclei have been suggested to be closely involved in cardiorespiratory activity through a chemoreceptor reflexogenic action.

The aim of this work was to illustrate a case of sudden unexplained late fetal death at $40+^{3}$ weeks of pregnancy. The anatomopathological protocol included, in particular, the analysis on serial sections of the cardiorespiratory autonomic nervous system, besides the in-depth examination of the placental disk, umbilical cord and membranes.

Through histopathological examination of the brainstem, hypoplasia of the arcuate nucleus and of the pre-Bötzinger complex was detected. These alterations were in association with severe third degree chorioamnionitis and vasculitis of the umbilical vein and amniochorionic vessels. While chorioamnionitis alone cannot be the cause of sudden death [10], it can allegedly contribute to it through cytokine release [11], which can enhance any chemoreceptor dysfunction as a major predisposing or contributing lethal factor.

\section{CASE REPORT} fetus.

It refers to the stillbirth of a $40+^{3}$ week pregnancy male

From the medical and gynaecological history file, we learned that the mother - a low risk primiparous woman with a normal pregnancy - was hospitalized for premature membrane rupture with onset of irregular uterine contractions. Sonography was performed showing that the fetus had a cephalic presentation fitting the pelvic inlet, and posterior placenta. Fetal heart beats (FHB) and active fetal movements 
were present. The woman entered labour, which took place regularly under obstetric control. FHB remained normal throughout labour, until expulsion of a stillborn baby (all resuscitation attempts failed).

\section{MATERIALS AND METHODS}

The postmortem examination required a careful study of all the organs, following the standardized anatomopathological procedures. The examination included an in-depth histopathological examination of the central autonomic nervous system (particularly of the brainstem centers checking the vital functions), of the cardiac conduction system, and of the fetal adnexa.

After fixation in $10 \%$ phosphate-buffered formalin, the brainstem was processed and embedded in paraffin. Transverse serial sections were made through the entire extension of the pons and medulla oblongata at intervals of $30 \mu \mathrm{m}$. For each level, twelve $5 \mu \mathrm{m}$ sections were obtained, two of which were routinely stained for histological examination using alternately hematoxylin-eosin and Klüver-Barrera stains. The remaining sections were saved and stained as deemed necessary for further investigations [12-14].

Fetal adnexa (placenta, umbilical cord, and membranes) were fixed in $10 \%$ buffered formalin and sampled in compliance with the protocol of the Institute of Pathologic Anatomy of Genoa [15] and then automatically processed, by using vacuum cycles of at least one hour per station, with at least two passes of xylol and two paraffins. These specimens were then cut with microtome into $3 \mu \mathrm{m}$ thick slices that were then stained, for screening, with hematoxylin-eosin, PAS (Periodic Acid Schiff), Perls (for iron), and Von Kossa (for calcium).

\section{PATHOLOGICAL FINDINGS}

Twenty-eight year old primiparous mother, with uneventful pregnancy and personal and family medical history which was negative for alcohol, and exposure to toxic substances. As to smoking, the mother had never smoked, while the father smoked 20 cigarettes a day; positive anamnesis for drugs during pregnancy (the mother took antibiotics Augmentin- for 6 days during $20^{\text {th }}$ week of pregnancy for treatment of enterococcus cystitis, which had been detected by urine culture, repeated again at week 36 and found to be more or less untreated).

At week $40+^{3}$ of pregnancy, premature membrane rupture occurred. Following examination of fetal adnexa, the umbilical cord was found to be $59 \mathrm{~cm}$ long, with 3 vessels (2 arteries and a vein), with $2 \mathrm{~cm}$ and $0.9 \mathrm{~cm}$ max and min diameter, respectively, and central insertion on the chorionic disc at $6 \mathrm{~cm}$ of distance from the maternal-fetal margin. The membranes, with regular insertion on the chorionic disc, look mat and thickened. The oval shaped chorionic disc is $686 \mathrm{gr}$ in weight, and $23 \times 17 \mathrm{~cm}$ in size, with $2.5 \mathrm{~cm}$ and 1.5 $\mathrm{cm}$ maximum and minimum thickness, respectively.

Following histological examination, the placenta showed signs of long-term hypoxic distress, with severe grade 3 chorioamnionitis (Fig. 1) and vasculitis of the umbilical vein and amniochorionic vessels.

Post-mortem examination: male fetus, $40+^{3}$ week gestational age, $3.929 \mathrm{gr}$ in weight and $55 \mathrm{~cm}$ crown-heel length,
$38 \mathrm{~cm}$ crown-rachis length, $32 \mathrm{~cm}$ occipital-frontal circumference, $11 \mathrm{~cm}$ occipital-frontal diameter, $9 \mathrm{~cm}$ biparietal diameter, $33 \mathrm{~cm}$ thoracic circumference, and 30.5 abdominal circumference; the foot is $8.2 \mathrm{~cm}$ long, the hand $6.5 \mathrm{~cm}$. A $3.5 \mathrm{~cm}$ long cord segment is annexed to the fetus; no external malformations were evident. When opened, the viscera were found to be in their right place, with no shape or structure abnormalities. Subpleural petechiae in the visceral pleura of both the right and the left lung were detected.

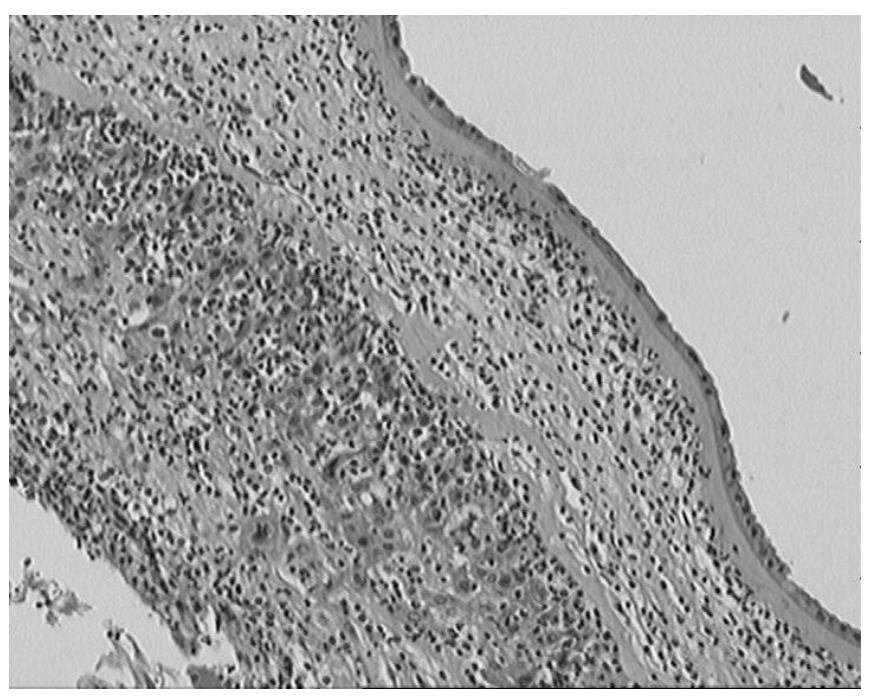

Fig. (1). Photomicrograph of severe grade 3 chorioamnionitis. Hematoxylin-eosin stain. Magnification: 20x.

At examination, a purulent exudate was observed in the bronchial-alveolar lumen, as well as pulmonary hypoplasia according to RAC (Radial Alveolar Count) microscopic criterion: 3.2 (n.v. >4.4) [16].

Histologic examination on serial sections of the brainstem showed severe hypoplasia of the arcuate nucleus (Fig. 2), as well as hypoplasia of the reticular formation and in particular of the pre-Bötzinger complex (Fig. 3). No alteration was found in other important vital centers (parafacial nucleus, locus coeruleus and parabrachial/Kölliker-Fuse complex in the pons and mesencephalon; hypoglossus, dorsal motor vagal, tractus solitarius, ambiguus, inferior olivary nuclei in the medulla oblongata). Fig. (4) shows a schematic representation of brainstem histological sections where the main vital centers are located.

Histologic examination of the conduction system showed a regular development of the sinus-atrial node. The atrialventricular node was fragmented, while islets of junctional tissue and resorptive degeneration were observed in the corpus fibrosum. The His bundle was fragmented, with fibrous shoots interspersed across the bifurcation.

\section{DISCUSSION}

As is well known, stillbirth can be caused by many different pathologies: in addition to maternal diseases, malformations, genetic and/or metabolic defects and abnormalities of fetal adnexa play an important role. Moreover, more than $60-80 \%$ of stillbirths are unexplained, mainly due to failure to carry out a complete, appropriate post-mortem investigation. 


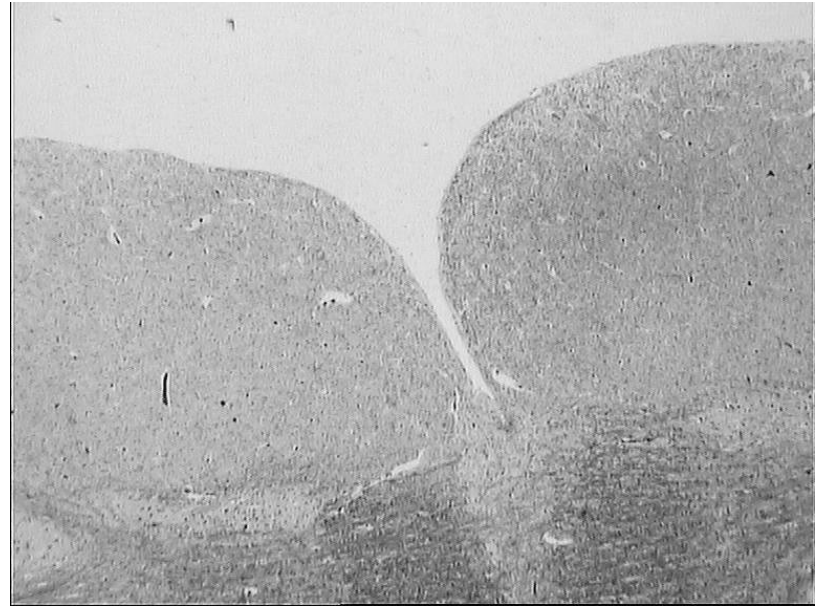

Fig. (2). Photomicrograph of a partial histological section of medulla oblongata at obex level showing severe bilateral hypoplasia of the arcuate nucleus. Klüver-Barrera stain. Magnification 20x.

Histological examination performed on a case of late unexplained stillbirth, object of this study, showed significant placental inflammatory alterations (severe, grade 3 chorioamnionitis, vasculitis of the umbilical veins and of the amniochorionic vessels) together with congenital development defects of important brainstem structures (severe hypoplasia of the arcuate nucleus, as well as of the reticular formation and, in particular, of the pre-Bötzinger complex) and with lung hypoplasia.

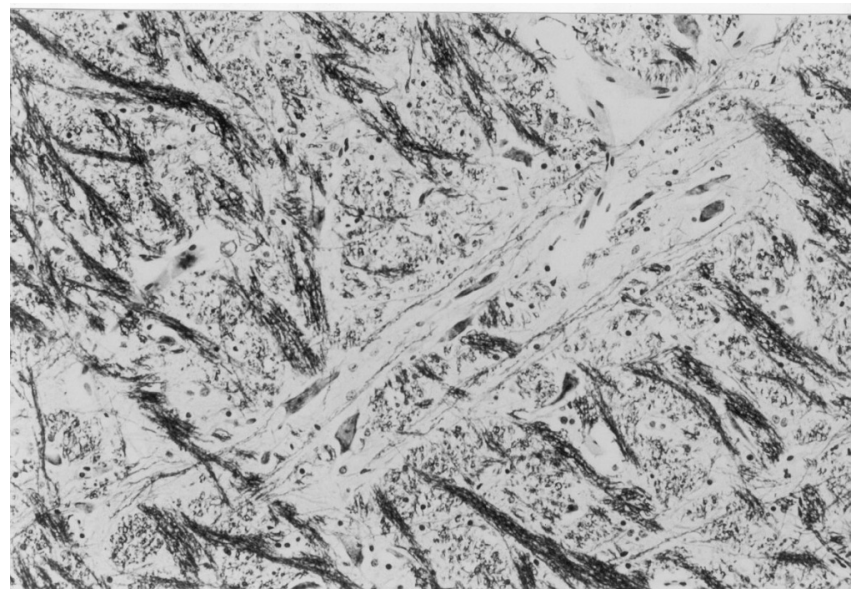

Fig. (3). Photomicrograph of a partial histological section of medulla oblongata showing hypoplasia of the pre-Bötzinger complex with lack of dendrites and flattened neuronal cell bodies. KlüverBarrera stain. Magnification 40x.

A careful diagnostic assessment is required in case of perinatal infections. Some cases are characterized by dramatic onset, which is therefore easy to diagnose at birth (preterm delivery, prolonged rupture of membranes, etc) [17, 18]. Conversely, few symptoms are present in other cases; therefore accurate laboratory, blood, microbiological and instrumental assessments are more than ever necessary. Infections - both ascending ones and blood infections - can quickly colonize the fetus causing its death [19]. In the case of ascending infection, germ propagation occurs through the vagina, with subsequent chorioamnionitis, and later penetra-

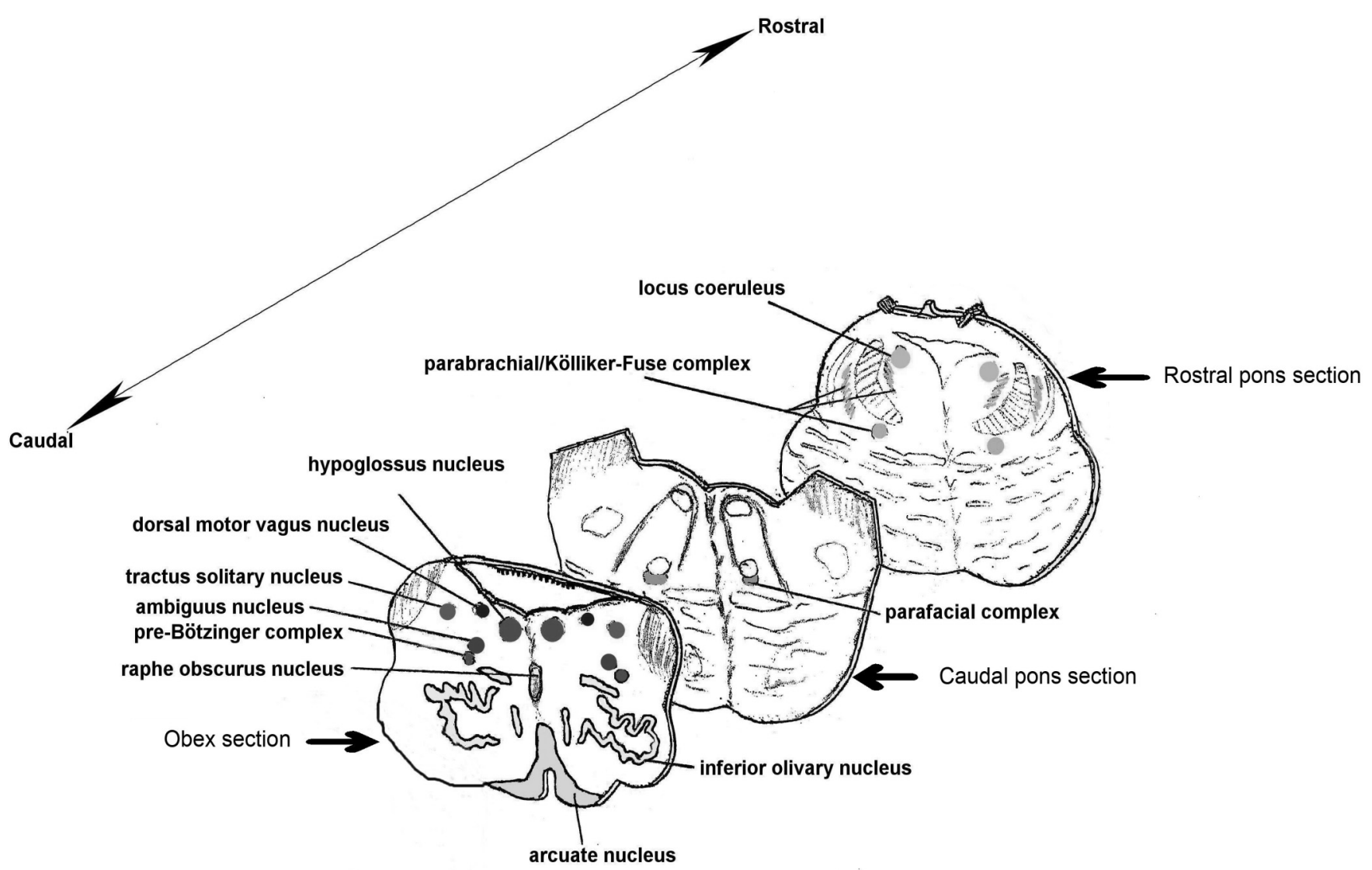

Fig. (4). Schematic representation of the main histological sections obtained from the brainstem for the histopathologic examination. 
tion into the fetus through suction of infected amniotic fluid, and then propagation from the mouth to the bronchi and the lungs, where endoalveolar infection develops. From the stomach, it then moves down to the small and large intestines; in the intestinal lumen, the so called "septic bolus" can be observed, consisting of granulocytes and exfoliating epithelial cells. Conversely, when the infection is spread through the blood from endometritis or, later, villitis foci, it penetrates the fetus by following the umbilical pathway, then propagating into the fetal arterial system and, in particular, into the aorta and renal arteries, where it triggers severe interstitial nephritis, with vasculitis and perivasculitis [10].

All in all, the inflammation picture of the adnexa found in the case here reported at post mortem examination cannot be held responsible for the sudden death. The inflammatory infiltrate had allegedly worsened the functional conditions already compromised by extended congenital brainstem abnormalities, namely by arcuate nucleus and pre-Bötzinger complex hypoplasia.

The arcuate nucleus is a component of the ventral surface of the medulla oblongata closely implied in cardiorespiratory activity, through its chemoreceptor reflexogenic action [6]. The pre-Bötzinger complex has been physiologically defined as a group of neurons of the ventrolateral medulla, that are essential for generating the respiratory rhythm, as well as for modulating eupneic breathing after birth [7, 20]. These two nuclei, obviously, in utero does not physiologically intervene in ventilatory chemoreflexogenesis but will become immediately operative at birth, with vital-lethal implications intra- and post-partum, as respiratory centers able to coordinate the pulmonary motor responses to hematic oscillations of $\mathrm{pO}_{2}, \mathrm{pCO}_{2}$ and $\mathrm{pH}$. Hypoplasia of both the arcuate and pre- Bötzinger nuclei can however lead to disturbances in the development of the respiratory apparatus, in particular to pulmonary hypoplasia, such as we observed in the present case. In fact, a low frequence of breathing movements which favour lung development occur in utero, mediated by brainstem vital centers [21].

Inflammatory processes of the adnexa, through cytokine release, had made chemoreceptive dysfunction more severe by triggering lethal reflexes [11].

In conclusion, this work aims to stress the importance of thorough investigations of both the autonomic nervous system and the placenta, in cases of sudden fetal death.

\section{ACKNOWLEDGEMENTS}

This study was supported by the Italian Lombardy Region target project n. 49210/2000 (Program of research and intervention for the reduction of the risk of SIDS and unexpected fetal death), by the agreement Lombardy RegionPfizer Italia S.r.1. n. 814/2006, and by Ministry of Foreign Affairs (joined projects of particular relevance "Anatomopa- thologic and genetic study of the unexplained perinatal death and SIDS") n. 269/P/0085087/2004 and n. 0083227/20006.

\section{REFERENCES}

[1] World Health Organization. International statistical classification on diseases and related health problems, $10^{\text {th }}$ revision (ICD-10). Geneva, World Health Organization 1993

[2] World Health Organization (WHO) Neonatal and perinatal mortality: Country, Regional and Global Estimates. 2006; pp 1-75.

[3] Cotzias CS, Paterson-Brown S, Fisk NK. Prospective risk of unexplained stillbirth in singleton pregnancies at term:population based analysis. BMJ 1999; 319: 287-88.

[4] Matturri L, Lavezzi AM. Pathology of the central autonomic nervous system in stillbirth. Open Ped Med J 2007; 1: 1-9.

[5] Ottaviani G, Matturri L. Histopathology of the cardiac conduction system in Sudden Intrauterine Unexplained Death (SIUD). Cardiovasc Pathol 2008 (in press).

[6] Matturri L, Minoli I, Lavezzi AM, Cappellini A, Ramos S, Rossi L. Hypoplasia of meduallary arcuate nucleus in unexpected late fetal death (stillborn infants): a pathologic study. Pediatrics 2002; 109: E43

[7] Lavezzi AM, Matturri L. Functional neuroanatomy of the human pre-Bötzinger complex with particular reference to sudden unexplained perinatal and infant death. Neuropathology 2008; 28: 10-6.

[8] Lavezzi AM, Ottaviani G, Ballabio GM, Rossi L, Matturri L. Preliminary study on the cytoarchitecture of the human parabrachial/Kölliker-Fuse complex with reference to sudden infant death syndrome and sudden intrauterine unexplained death. Pediatr Dev Pathol 2004; 7: 171-9.

[9] Lavezzi AM, Ottaviani G, Rossi L, Matturri L. Hypoplasia of the parabrachial/Kölliker-Fuse complex in perinatal death. Biol Neonate 2004; 86: 92-97.

[10] Fulcheri E, Bulfamante G, Resta L, Taddei GL. Embryo and fetal pathology in routine diagnostics: what has changed and what needs to be changed. Pathologica 2006; 98: 1-36.

[11] Matturri L, Ottaviani G, Ramos SG. Discrete T- lymphocytic leptomeningitis of the ventral medullary surface in a case of sudden unexpected infant death. Adv Clin Pathol 1998; 2: 313-16.

[12] Matturri L, Ottaviani G, Alfonsi G, Crippa M, Rossi L, Lavezzi AM. Study of brainstem, particulary the arcuate nucleus, in sudden infant death syndrome (SIDS) and sudden intrauterine unexplained death (SIUD). Am J Forensic Med Pathol 2004; 25: 44-48.

[13] Matturri L, Ottaviani G, Lavezzi AM. Techniques and criteria in pathologic and forensic-medical diagnostics of sudden unexpected infant and perinatal death. Am J Clin Pathol 2005; 124: 259-68.

[14] Matturri L, Ottaviani G, Lavezzi AM, Guidelines for neuropathologic diagnostics of perinatal unexpected loss and sudden infant death sindrome (SIDS). A technical protocol. Virchows Arch 2008; 452: 19-25.

[15] Fulcheri E, Grillo F, Musizzano Y. Il trattamento della placenta per l'esame istopatologico finalizzato allo studio ed alla diagnostica del danno neurologico feto-neonatale. Riv It Ost Gin 2006; 9: 475-81.

[16] Matturri L, Lavezzi AM, Cappellini A, Rossi L. Association between pulmonary hypoplasia and hypoplasia of arcuate nucleus in stillbirth. J Perinatol 2003; 23: 328-32.

[17] Goldenberg RL, Thompson C. The infectious origins of stillbirth Am J Obstet Gynecol 2003; 189: 861-73.

[18] Kalousek DK, Bmford S. Amniotic rupture sequence in previable fetus. Am J Med Genet 1988; 31: 174.

[19] Bittencourt AL, Garcia AG. Pathogenesis and pathology of hematogenous infections of the fetus and newborn. Pediatr Pathol Mol Med 2002; 21: 353-99.

[20] Rekling JC, Feldman JL. PreBötzinger complex and pacemaker neurons: hypothesized site and kernel for respiratory rhythm generation. Annu Rev Physiol 1998; 60: 385-405.

[21] Boddy K, Dawes GS. Fetal breathing. Br Med Bull 1975; 31: 3-7. 